\title{
Le collège, la ville, et la mixité sociale : La fabrique de la distribution
} des élèves

\section{Laurent Visier, Geneviève Zoïa}

\section{Résumé}

Cet article explore l'enjeu d'une politique d'égalité scolaire qui, après la période de rationalisation et d'unification de l'offre, s'inscrit dans une perspective de mélange et de diversification des publics au sein des établissements. Si l'évitement par les parents de certains établissements scolaires est incontestable, l'affirmation d'une intention séparatiste des acteurs sociaux fonctionne comme un obstacle pour penser une politique de mixité culturelle et sociale. Les inégalités de l'offre sur les territoires urbains et le ressenti des parents, décrits ici dans le cadre d'une agglomération de 500000 habitants, doivent être pris au sérieux si l'on veut envisager une politique effective de mixité.

\section{Citer ce document / Cite this document :}

Visier Laurent, Zoïa Geneviève. Le collège, la ville, et la mixité sociale : La fabrique de la distribution des élèves. In: Les Annales de la recherche urbaine, N¹06, 2010. Mélanges. pp. 38-47;

doi : https://doi.org/10.3406/aru.2010.2781

https://www.persee.fr/doc/aru_0180-930x_2010_num_106_1_2781

Fichier pdf généré le 23/04/2018 


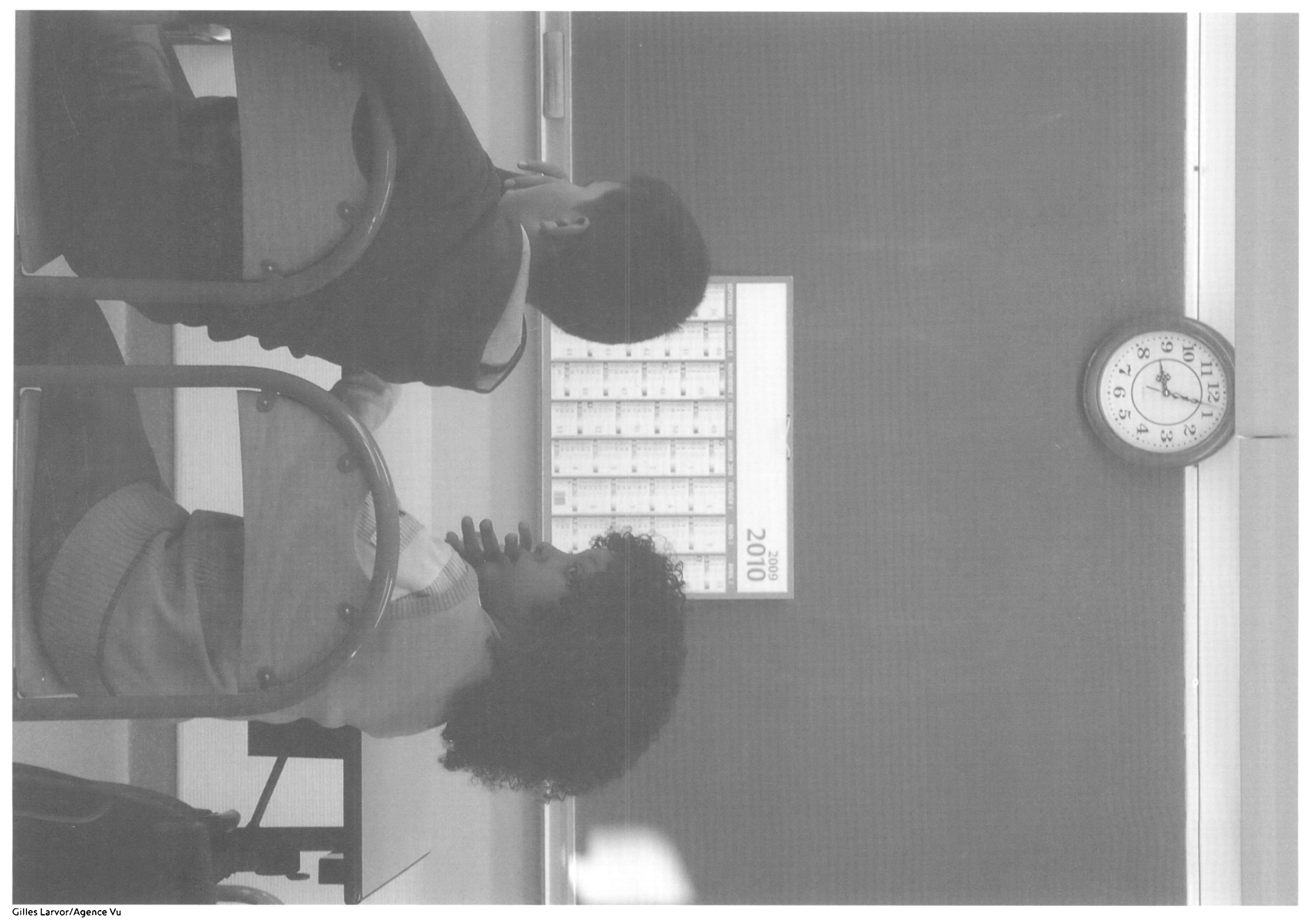




\section{Le collège, la ville, et la mixité sociale*}

La fabrique de la distribution des élèves

\section{Laurent Visier \\ Geneviève Zoïa}

L'heure est à la reconnaissance de l'inégalité de l'offre entre les établissements du territoire français d'éducation (Maurin, 2004). Non seulement les inégalités indexant la réussite scolaire à l'origine sociale que les sociologues ont largement analysées depuis les années soixante, mais aussi celles produites par la composition des publics des établissements inscrits dans des territoires. L'effet school mix ou effet de voisinage analysé depuis de nombreuses années (Coleman, 1966) a un impact sur les parcours scolaires non négligeable. Autrement dit, malgré l'unification du système d'enseignement en une filière unique jusqu'à la fin du collège, et aussi brutale que paraisse cette proposition, chaquc ćlève ne bénéficie pas du même service d'éducation et donc des mêmes chances, parce qu'il ne fréquente pas les mêmes pairs.

On ne sortira pas facilement de ces difficultés, et certainement pas seulement par la recherche de boucs émissaires, parents consommateurs, citoyens égoïstes ou promoteurs cupides. La réalité montre que ces nouvelles inégalités ne peuvent être expliquées par des infractions à la règle, ni combattues par des injonctions en ce sens. On n'en sortira pas non plus par des pseudo certitudes prononcées sur un ton d'évidence selon lesquelles peu importerait le lieu de scolarisation puisque chacun réussirait in fine selon ses capacités. Du reste, si tel était le cas, pourquoi un telle polarisation sur les questions de carte scolaire, de choix de l'école et de contournements ? C'est à la question de la justice que renvoie celle de la mixité sociale à l'école. Nos travaux sur les choix scolaires des familles nous enseignent combien les parents de tous milieux sociaux se montrent sensibles à ce sujet (Visier, Zoïa, 2008) : où commence et où s'arrête leur responsabilité ? Comment pensent-ils contribuer à l'échec ou au succès de leurs enfants ? Comment distinguer talent, travail et mérite ? Les parents vivent mal la contradiction entre des principes d'égalité auxquels ils tiennent, et les injustices auxquelles ils contribuent en choisissant un établissement. Pourtant, s'il y a bien contradiction, celle-ci n'est pas réductible à une hypocrisie : le bon parent est à la fois soucieux du bien de son enfant et de l'égalité sociale. Le principe de différence (Rawls, 1971) selon lequel des inégalités sont acceptables lorsqu'elles sont à l'avantage des défavorisés est largement partagé, mais la reconnaissance de l'arbitraire de la distribution du talent ne va pas jusqu'à remettre en cause la légitimité de ce dernier. Il reste que les parents sont très sensibles à ces nouvelles inégalités que constituent les expériences de scolarisation si différentes.

Pour comprendre la construction des pratiques des familles quant à la scolarisation de leurs enfants, nous nous attacherons dans un premier temps au statut moral accordé aux contournements scolaires, nous synthétiserons ensuite la tonalité sociale des établissements, nous envisagerons enfin les propos des professionnels (enseignants ct principaux) ainsi qu'au temps fort de mise en œuvre d'une politique de distribution des publics qu'est la commission de dérogation, avant de revenir aux ressorts de l'action des familles sur le choix du collège.

\footnotetext{
"Nous dévcloppons ici quelques-uns des résultats de la recherche " Mixité sociale au collège * réalisée de 2006 à 2008 dans le cadre du programme "Renouveler l'urbain au nom de la mixité ? ", Puca, ministère de l'Écologie, du développement et de l'aménagement durable et cofinancée par l'ACSE Languedoc-Roussillon.
} 


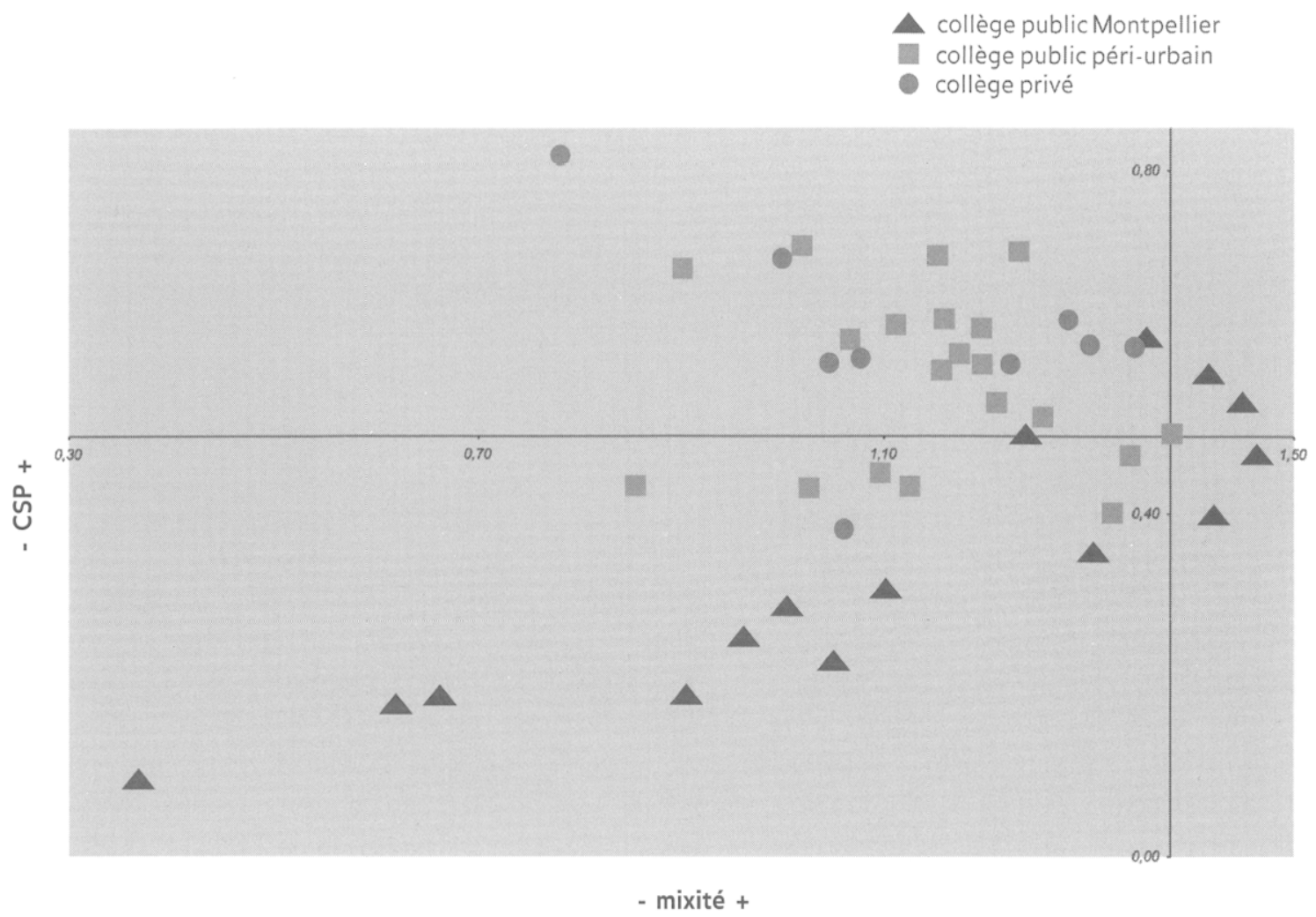

\section{Les parents sont-ils coupables d'exercer des choix?}

Nos entretiens révèlent l'existence d'un très fort sentiment d'opacité de l'univers scolaire : les parents ne se sentent ni acteurs ni partenaires du système; tout au plus se perçoivent-ils capables de stratégies selon leur degré d'initiation ou de familiarité avec le monde scolaire. On peut mesurer, à cet égard, la concentration des analyses critiques sur cette question des stratégies parentales. Ces analyses sociologiques présupposent un conflit entre intérêt général et individuel, et intentent un procès méthodique en séparatisme à l'encontre de la moindre stratégie identifiable. Une première vague de ces travaux, dans les années quatre-vingt (Ballion, Oeuvrard, 1991, Caillé, 1993) a défini les comportements des parents à l'école comme consuméristes. D'autres explorent aujourd'hui la clôture sociale ou l'entre soi (Van Zanten, 2009 ; Oberti, 2007). Les classes moyennes ou supérieures fuiraient la différence sociale et ethnique, organisant, de fait, des situations d'apartheid scolaire. L'intention de la ségrégation ne fait pour ces auteurs aucun doute : M. Oberti s'interroge sur l'identité des responsables des processus ségrégatifs, pour lui les classes sociales supérieures, alors que pour A. Van Zanten, ce sont plutôt les classes moyennes. E. Maurin avance l'idée d'un processus généralisé de sécession. En conséquence de ce débat sur la plus ou moins grande responsabilité de telle ou telle classe ou de toutes, la carte scolaire apparait d'ailleurs fréquemment comme la seule politique vertueuse face aux désordres que provoquerait sa disparition.

Il est pourtant possible de sortir d'un raisonnement qui présente l'intérêt général comme mis en échec par l'égoïsme des parents consommateurs : affirmer que les profils sociaux, les espaces urbains et les pratiques d'évitement se correspondent relève d'une pensée tautologique montrant que les classes supérieures optent pour des espaces résidentiels sélectifs, qu'elles ont plus recours à la scolarisation hors secteur que les classes moyennes, lesquelles y ont plus recours que les classes populaires. Ces processus ne sont pas contestables, mais constituent un point de départ plus qu'unc explication. Car considérer l'attitude des parents à travers l'idée morale de séparatisme social ne restitue pas la complexité de leurs motivations. Si les formes et les effets de "sécession » sont bien réels, l'affirmation d'une intention séparatiste des acteurs sociaux fonctionne surtout comme un obstacle pour penser une politique de mixité culturelle et sociale. Il convient plutôt de raisonner à partir des inégalités de l'offre et de leur distribution territorialisće pour analyser ensuite l'action des parents, si l'on veut pouvoir envisager ce que pourrait être une politique de mixité. En effet, ce qui différencie les propos des parents ne tient pas avant tout à leur position sociale mais à leur lieu de résidence, c'est-à-dire à leur regard par rapport au collège de proximité. 


\section{Configuration de la distribution des élèves dans une agglomération de $\mathbf{5 0 0} 000$ habitants}

Les propos des parents et des professionnels ne peuvent être lus sans considérer la composition sociale des différents collèges. L'enquête que nous avons menée pendant deux ans dans une agglomération de 500000 habitants fait apparaître non pas seulement deux pôles mais bien trois grandes figures territoriales dans la distribution des élèves selon la catégorie sociale de leurs parents. Nous parlons de mixité sociale pour caractériser la capacité de chaque collège à scolariser ensemble des enfants issus de catégories sociales contrastées et nous différencions cette notion de celle de moyenne sociale qui décrit la tonalité sociale de l'établissement (graphique) ${ }^{1}$.

La première figure est celle du ghetto. La mauvaise réputation des collèges y est liée à leurs quartiers de recrutement, socialement très défavorisés. Si certains responsables académiques et professionnels du système éducatif parlent néanmoins de mixité à propos de ces quartiers, c'est le fait d'une tendance à assimiler mixité et pauvreté ou bien à désigner par mixité la multiplicité des origines ethniques. Nos travaux, confirmant en cela de nombreux autres, montrent dans ces quartiers non pas une mixité sociale mais au contraire une homogénéité des catégories sociales les plus basses. Les catégories moyennes, mais aussi beaucoup de familles défavorisées, lorsqu'elles en trouvent les moyens, fuient ces collèges'.

La deuxième est celle des établissements publics du périurbain ainsi que des collèges privés du centre-ville. Ces dcux types se ressemblent en effet étrangement quant à la composition des publics reçus. Le choix résidentiel et celui de l'école n'apparaissent donc pas de nature distincte. Que ce soit par l'un ou par l'autre, une grande part de la population fait en sorte que ses enfants soient scolarisés « au bon endroit », c'est-à-dire dans un milieu considéré comme acceptable. La mixité dans ces établissements est souvent faible.

La troisième est celle des collèges publics de centre-ville : loin des clichés qui les assimilent à un « entre soi » des familles de haut niveau social, les collèges publics du centre-ville regroupent des élèves issus de l'ensemble des catégories sociales. Ils montrent ainsi que la mixité sociale n'écuivaut pas à une perte d'attractivité de l'établissement et démontrent surtout qu'une image positive et une mixité forte peuvent aller de pair. La vieille opposition entre établissements privilégiés

1. Sur ce graphique, la position de chaque collège de l'agglomération est caractérisée en ordonnée par un niveau social moyen (movenne des CSP) et en abscisse par la mixité sociale (variance des CSP). (Zoïa, Visier, 2007).

2. On retrouve à Montpellier le ghetto scolaire décrit dans l'académie de Bordeaux (Felouzis, 2005). Si nous avons exclusivement travaillé la dimension sociale (CSP), la dimension ethnique de la ségrégation est également très forte. du centre-ville et de seconde zone en périphérie n’est plus de mise. La ville se recompose et l'école l'accompagne, notamment dans des agglomérations de la taille de celle que nous avons étudiée. Le fait que l'aire urbaine étudiée ait connu une croissance démographique importante au cours des demières décennies permet de penser que s'opère une redistribution des cartes à laquelle la plupart des analyses sociologiques, souvent focalisées sur la situation francilienne, ne rendent guère justice.

\section{Les professionnels : reconnaître les situations d’inégalité}

Face à ce constat d'une hétérogénéité des publics selon les collèges, les professionnels de l'Éducation nationale affirment comme un préalable l'équivalence des établissements. Cela ne signifie pas que les enseignants ou les chefs d'établissement soient schizophrènes ou hypocrites, mais qu'ils tentent de maintenir l'idée d'un système unique tout en constatant des situations empiriques très contrastées. L'affirmation de l'unité du système revient, pour chacun, à dire que son propre établissement relève d'une situation normale. Ainsi, lors des réunions dans les écoles pour présenter son collège aux parents, le principal d'un établissement socialement très défavorisé affirme que « celui-ci n'est pas plus mauvais que les autres et qu'il tient à le montrer ». Assistant à l'une de ces rencontres, nous l'entendons en effet décrire la richesse d'une offre scolaire particulièrement développée (langues, sports, activités...), mettre en avant un cadre physique attrayant et enfin insister sur ce terme d' " excellence ", dont usent volontiers les chefs des établissements scolarisant les catégories sociales les plus basses. À l'autre bout du spectre dans les établissements de milieu social élevé, il est convenu de souligner qu' "on n'a pas ici que des bons élèves " ou qu' « il ne faut pas croire qu'on est une école de privilégiés ». Lorsque nous commentons la liste des élèves et des professions des parents, ces dirigeants consentent à l'évidence, mais comme une « coïncidence cette année ».

Bref, les directeurs d'école et les principaux de collège insistent sur le caractère ordinaire de leur établissement, marquant ainsi leur adhésion à la valeur d'égalité qui structure le discours sur l'école publique depuis un siècle et demi : une école ou un collège «normal » se doit de scolariser toute la palette des situations sociales existantes et euxmêmes se sentiraient en défaut si ce n'était pas le cas.

En revanche, lorsque les mêmes professionnels sont questionnés en tant que parents d'élèves, le réalisme prend le dessus : " pour mes grands qui ont plus de vingt ans aujourd'hui, la question de la scolarisation sur l'école du quartier ne se posait pas, ça allait de soi ; mais pour le petit dernier qui rentre en sixième, ce n'est plus possible. Je suis bien placée pour savoir que s'il est inscrit ici, il n'a pas les mêmes chances que dans un établissement hors du quartier ». Que la même 


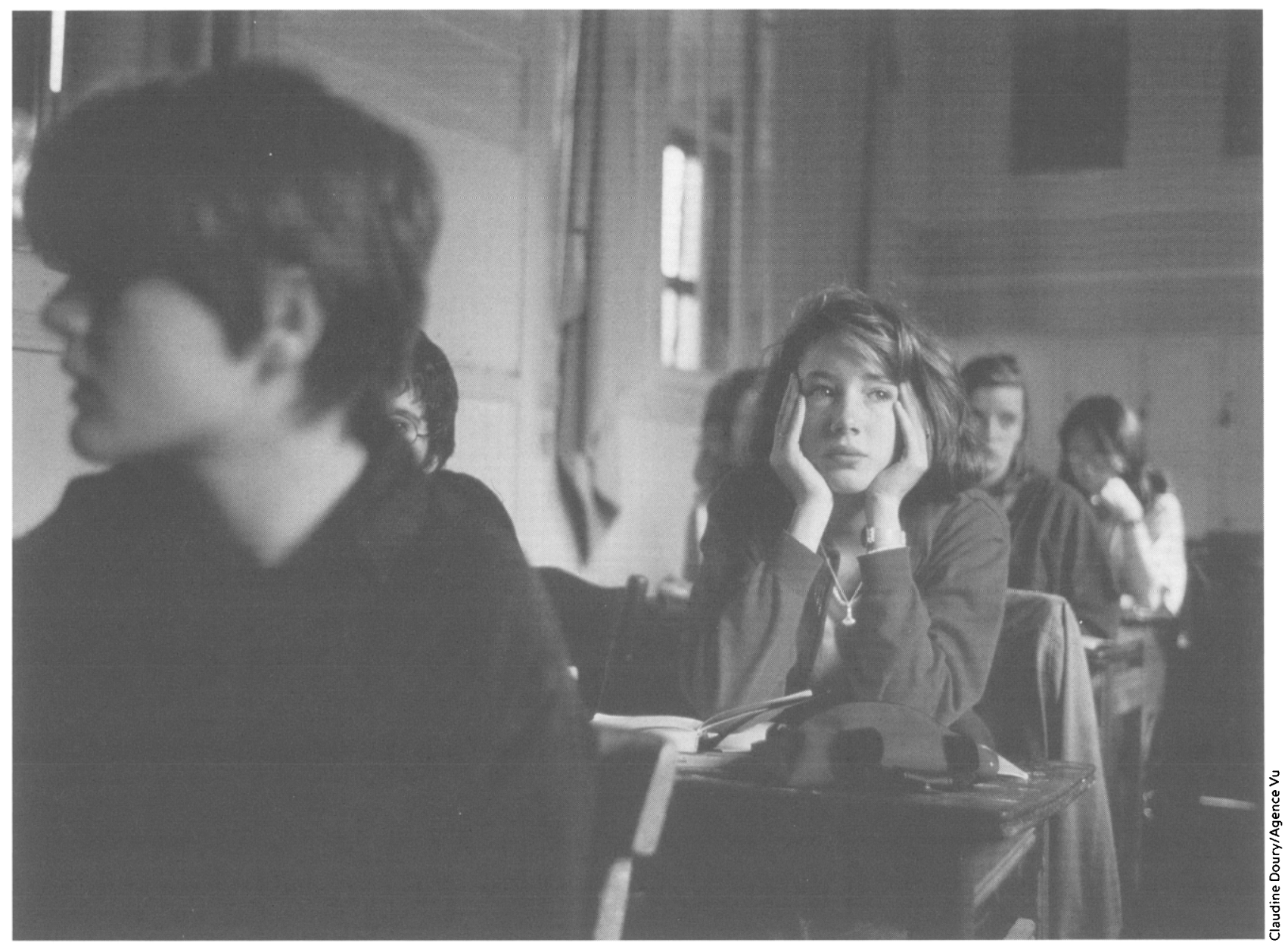

personne parle différemment comme professionnel et comme parent ne doit pas être analysé comme une marque de duplicité mais comme celle d'un conflit irrémédiable de positions, conflit d'autant plus aigu que l'on réside " au mauvais endroit ». En effet, les seuls qui ont à faire ce grand écart entre leurs valeurs et leurs pratiques sont ceux dont la résidence n'est pas sectorisée sur un établissement conforme à leurs attentes.

La même contradiction apparaît entre l'offre scolaire et la demande d'éducation. Quelle que soit l'offre proposée (en enseignants, locaux, projet), elle s'avère au final surdéteminée par les élèves accueillis. Les nuênes enseignants qui proclament en préalable l'unité de l'école républicaine illustrent ensuite l'impact des publics sur l'offre : «On est devenu une école de pauvres et d'immigrés. Ça ne sert à rien de vouloir faire revenir les catégories moyennes du quartier ici. Ils ne sont pas fous. Quand on me demande un certificat de radiation, ça m'énerve vraiment comme directrice, mais comme parent je ne peux pas faire autrement que de penser qu'ils ont raison de partir d'ici ». D'un côté, il y a la règle commune et les intérêts professionnels : « des enfants qui s'en vont, c'est moins de moyens, moins de temps de décharge pour le directeur, moins de prime. En tant que directeur, il ne faut pas perdre d'élève et surtout ne pas perdre de classe ». De l'autre, il y a une exigence de justice : « c'est pas possible de laisser ce gamin ici. Il va se faire bouffer ». Que les enfants d'enseignants soient les premiers à éviter les établissements les plus difficiles peut donc être interprété autrement que comme un délit d'initié. Bien sûr, les professionnels se repèrent mieux dans les codes des différentes filières de dérogation, mais ils savent surtout, mieux que quiconque, que les établissements ne sont pas équivalents. Ils ne sont prisonniers ni de « réputations " ni de « rumeurs »: ils agissent en connaissance de cause.

Au-delà de la distribution des publics dans les collèges, reste la politique de composition des classes. Si ses acteurs, chefs d'établissements, enseignants, affirment toujours ne pas faire de classe de niveau, c'est souvent un déni de pure forme. Ils concèdent assez vite qu' "évidemment les classes ne sont pas identiques. Mais on essaie de ne pas mettre tous les bons dans une et tous les mauvais dans l'autre ". L'expression "classe de niveau " recouvre une réalité aux formes multiples, options langues, sports, musique et les classements trop lisibles sont évités. La palme du déni revient à la classe internationale du grand collège de centre-ville, unanimement reconnue par les parents comme " classe de choc » et par les élèves comme " classe d'intellos », et qui a pour nom... « sixième zéro ». Les chefs d'établissement commentent ce travail de composition en 
présentant les contraintes antagonistes qu'ils conjuguent : " personne ne peut dire qu'on va constituer des classes absolument identiques. Ce n'est pas jouable. Si je dis aux parents que je réussis à garder ici qu'ils vont être mélangés avec les plus mauvais élèves, ils s'en iront ». Ainsi la relation entre mixité des établissements et mixité des classes constitue bien un enjeu, les déclarations de principe sur l'égalité des classes à l'intérieur d'un établissement pouvant même jouer contre l'égalité des établissements.

\section{Organiser la mixité sociale sur le territoire : une journée à la Commission de dérogation}

Pour les inscriptions en sixième, les commissions de dérogation réunissent l'ensemble des principaux des collèges publics du bassin de formation autour du chef de la direction concernée à l'Inspection académique. L'objectif consiste à réguler des flux, le peuplement des établissements et l'adéquation du nombre d'élèves au nombre de divisions pour l'année suivante. Les demandes familiales y sont abordées ensuite. Cette réunion débute ainsi par un rappel à l'ordre en direction des chefs d'établissement, relatif à leur tendance à la surévaluation des besoins.

Elle se déroule à l'aide de la carte de la ville découpée en secteurs scolaires et des tableaux synthétisant la taille des établissements, le nombre de divisons et le nombre d'élèves : "n'oublions pas que notre réunion a d'abord pour but de gérer les équilibres ». Chaque collège est traité en fonction du nombre de places disponiblcs ct du nombre de demandes de départ et d'arrivée des élèves. Cet examen donne lieu à des négociations entre l'IA, l'établissement de secteur et l'établissement demandé : « il y a quinze demandes vers $\mathrm{X}$, ça ne pose pas de problème. Il y a de la place. On peut les prendre. En revanche les six qui viennent de $Z$, ce n'est pas possible, sinon on vide cet établissement. On ne peut pas laisser partir tout le monde ", déclare le chef de service.

Sont ensuite considérées les demandes des familles. Dans les secteurs nouvellement découpés, on considère que la règle doit s'appliquer de façon stricte, pour ne pas laisser se perpétuer des « traditions historiques qui réduiraient à néant le redécoupage ». Certains territoires sont placés sous le projecteur, comme celui de ce collège classé "Ambition Réussite ", et qui se trouve entouré d'établissements aux publics socialement plus élevés. Dans ce cas, l'objectif est clairement affirmé : faire un exemple en refusant les dérogations demandées par les parents pour orienter leurs enfants vers les autres secteurs. De nombreuses demandes de dérogation sont formulées, mais la nouvelle règle s'applique avec fermeté, au nom du motif de la mixité. "On vient de refaire la carte, si c'est pour céder tout de suite, ce n'est pas la peine ", affirme le responsable de l'Académie, suivi par les chefs d'établissement. Mais bien peu de familles se plieront ensuite à cette décision, préférant se rabattre sur le secteur privé pour fuir ce qu'elles ressentent comme une immense injustice.

Les demandes des parents connaissent un succès très inégal selon les motifs avancés. Les demandes à titre médical ou social, accompagnées d'attestations, sont les moins nombreuses et généralement acceptées. La case " convenance personnelle » est, à l'évidence, celle que les parents doivent éviter de cocher. Quand les parents mentionnent que leur enfant « est fragile » et qu'ils ont « peur pour lui », ou bien quand ils évoquent les « mauvaises fréquentations » ou la " mauvaise ambiance " auxquelles il s'exposerait dans le collège de proximité, à l'opposé du "bon cadre de travail » et du bon collège » vers lequel ils souhaitent diriger leur enfant, leurs chances sont quasi nulles. Pire, lorsque des parents mentionnent " les événements récents qui se sont produits dans l'établissement ", le chef de l'établissement en question va jusqu’à demander le nom de la famille concernée. Face à une demande avançant pour motif le « bon taıx de réussite de ce collège ", les chefs d'établissement s'écrient en chœur : "Qu'est-ce que c'est qu'un bon collège ? Il faudrait nous le dire".

Personne n'est dupe du fait que la « convenance persomnelle " traduit des motivations identiques à celles des demandes formulées plus efficacement dans la rubrique pédagogie qui traite de façon euphémisée des matières à option que l'on trouve dans les collèges les plus convoités. L'évocation du niveau différencié des établissements constitue un tabou : formuler une demande en ces termes revient à offenser l'Éducation nationale. Pourtant, les tractations entre les chefs d'établissements reposent clairement sur cette connaissance interne des inégalités de l'offre et de leur influence sur la carrière des élèves. Cet échange entre deux principaux de collèges à propos d'un élève qui demande une dérogation d'un collège moyen des faubourgs vers le collège de centre-ville le montre on ne peut plus crûment :

"- Non, c'est un bon élève. Celui-là, je le garde

- Allez écoute, ce gamin, il y a de la place à X, laisse le partir, il va peut-être aller en prépa un jour (responsable I.A.)

- Ah d'accord !... De chez moi, on ne va pas en prépa alurs?".

Chacun cherche à maintenir ses effectifs, à éviter de perdre des classes et à tirer sa composition sociale vers le haut. Cependant la commission de dérogation n'est pas non plus un dispositif implacable de reproduction sociale par l'acceptation des demandes des catégories socialement élevées; elle se présente surtout comme un outil de gestion des flux d'élèves visant à affiner à l'unité près l'adéquation des moyens aux effectifs. Dans le cadre de cette mission, la question de la mixité sociale est subsidiaire. Ainsi, face à un chef d'établissement qui résiste à l'affectation dans son collège d'une dizaine d'élèves plus défavorisés que son public habituel, le responsable de l'I.A. 
répond : " de toute façon, tu n'as pas tellement le choix. S'il y en a quarante qui partent de chez toi, je te fermerai des divisions, et on te remplira avec n'importe quoi. Donc tu pourrais les prendre ces demandes, ce sont des gens qui font au moins une démarche vers ton établissement ».

Ce type d'inégalités liées au voisinage engage une politique de mixité sociale qui ne peut être conçue comme un outil de rationalisation, à l'image de la planification de l'action publique réalisée dans les années soixante au grand moment de la massification. Face à cet enjeu nouveau et pressant des avantages ou des désavantages liés aux fréquentations de chacun, de quartier, d'établissement ou de classe, l'intérêt sociologique de la distinction entre " bonnes » et " mauvaises " motivations à déroger, entre dérogations " légitimes " et "stratégiques " (Felouzis et $a l$, 2005) parait bien faible. Pour comprendre les affirmations initiales de l'égalité des établissements par les professionnels aussi bien que leurs contorsions de discours ensuite ou même que les raisonnements sociologiques, il faut revenir à un point essentiel : la sectorisation est associée de façon quasi unanime à la logique de l'intérêt général (Combaz, 2003).

\section{Les familles : entre bien de l'enfant et idéal de justice}

Si le rapport des parents à la scolarité de leurs enfants est conditionné par le lieu de résidencc, la plupart d'entre eux racontent le moment du passage en sixième comme celui de la nécessaire duplicité. Il faut « faire l'imbécile », " utiliser des stratagèmes », « décrypter ». Qu'ils habitent ou non dans « le bon quartier », les logiques du collège sont toujours une épreuve, plus ou moins difficile selon les ressources dont chacun dispose. La dénonciation de leurs comportements comme mus par une logique de consommation n'a pas grand sens. Affirmons-le nettement : les parents sont tous des contourneurs potentiels. Certes, la majorité des élèves sont scolarisés dans le collège public de secteur. Mais il ne faudrait pas en déduire que le choix du collège de secteur serait un non choix et vaudrait acceptation de la règle. Aller dans le collège de secteur correspond le plus souvent à des options résidentielles préalables qui règlent la question. Le périurbain est exemplaire sur ce point ; le plus souvent le choix du collège ne s'y pose pas, alors que dans les villages sectorisés sur les collèges difficiles de la ville, celui-ci redevient une question essentielle. La logique du choix n'est donc pas propre à certaines catégories, mais elle est rendue visible dans certains territoires sensibles dans lesquels les parents jugent irrecevable la proposition du collège de secteur. Cependant, une analyse qui reste focalisée sur ce point manque l'essentiel : à savoir que les contournements sont intéressants, non pas parce qu'ils donneraient à voir quels parents seraient fautifs, mais pourquoi certaines situations sont jugées par ces derniers comme inacceptables ou injustes.

Tous les parents choisissent et pourtant tous affirment tenir à la mixité. Comment expliquer cette apparente contradiction? In élément de réponse est qu'ils tiennent plus encore au bien de leur enfant, même si chacun d'entre eux le définit de façon différente. Peut-on pour autant parler d'un fossé entre intérêt général et individualisıne? Ce serait tenir pour hypocrite la position des parents, alors même qu'ils prennent explicitement position en faveur de la mixité sociale. Selon quels arguments ? On peut ranger ceux-ci en trois catégories. Une première série de propos renvoie à l'idée d'une mixité émancipatrice. Les milieux populaires ont avantage à fréquenter les catégories moyennes et supérieures qui sont porteuses des valeurs de progrès et de la civilisation et de modèles culturels pouvant corriger la complaisance des milieux populaires vis-à-vis de leurs enfants. Un deuxième type d'argument de propos renvoie aux bienfaits de la mixité fonctionnelle définie à la fois par un juste équilibre entre composantes sociales interdépendantes et produisant un effet d'harmonie. C'est d'ailleurs cette juste proportion de la bigarrure sociale qu'illustre dans les propos la sectorisation scolaire idéale. Ces images de convivialité nient souvent une part des difficultés de la différenciation sociale, désignant des coupables sans lesquels tout fonctionnerait à merveille. Ainsi, dans un quartier d'immigration, un parent d'élève, qui a quitté les lieux affirme : " ici, il y a une poignée de gens qui font que ce n'est pas vivable, on les comnaîl ». Enfinı, une troisième série d'arguments renvoie à la mixité politique, au sens de la diversité culturelle comme confrontation organisée des différences. L'enjeu est alors celui du maintien de sociabilités complexes.

Du fait de cette contradiction entre l'idéalisation de la mixité sociale et une réalité moins idyllique, deux normes entrent en tension : celle du bon parent qui souhaite les meilleures conditions de scolarisation pour son enfant, et celle du bon citoyen promoteur d'égalité et de mixité sociale. Cette contradiction explique dans les entretiens les remarques mi-amusées mi-attristées, sur les justifications interminables qu'entraîne le lieu de scolarisation des enfants dans les conversations en famille ou entre amis. Ce père ingénieur raconte comment le choix du collège fut l'occasion d'échanges riches avec leur fils. « Il faut être au bon endroit, nous ne voulons pas prendre de risques, on lui a expliqué qu'il faut faire cet effort pour préserver ses choix futurs dans la vie ". Les parents savent que, pour construire le bien de leur enfant, le lieu de scolarisation est important, étant donné non seulement le temps que l'on y passe, mais aussi ce qu'on peut aujourd'hui escompter de l'école : réussite, mobilité, émancipation. Parler de consommation ou de marché scolaire constitue donc à la fois une évidence et un contresens. Une évidence car chaque parent a conscience de la différenciation des contextes de scolarisation et peut 
exercer des choix. Un contresens pourtant, car chacun ne visera pas toujours la classe de meilleur niveau dans l'établissement le plus haut de gamme, mais cherchera à ne pas être lésé, selon sa propre position.

Ce qui est recherché, ce n'est donc un établissement super performant, mais le collège " acceptable » pour l'enfant et les parents. Une majorité de parents s'interroge à la fois sur ce qui est à mettre en œuvre pour atteindre des situations pas trop injustes, et sur les limites morales de leurs entreprises.

\section{Ce que fuient les parents et ce qu'ils acceptent}

Quelles sont les raisons invoquées par les parents pour ne pas fréquenter le collège de proximité ? S'agit-il d'un mouvement généralisé qui conduirait chacun à se replier sur un monde toujours plus segmenté ? On peut affirmer que les parents veulent éviter de vivre un trop grand écart. Mais deux clarifications s'imposent. La première concerne leur intention : certes chacun demande à ne pas être noyé dans un monde social complètement différent du sien, à ne pas faire partie d'une minorité trop étroite. On peut bien sûr en déduire que cela contribue à la construction de lieux socialement homogènes, selon le modèle de la ségrégation développé dans les années soixante-dix par Shelling ${ }^{3}$. À partir de la théorie des jeux, ce dernier montre comment, sans qu'il y ait besoin de volonté ségrégative, le seul fait de refuser d'être trop minoritaire au sein du voisinage conduit mécaniquement à une structure de peuplement très homogène. Ainsi, constater empiriquement des formes d'entre soi, ou même de développement séparé ne permet pas d'inférer l'existence de volontés individuelles allant en ce sens. L'existence d'une forte concentration des élèves d'origine étrangère dans certains collèges ne signifie pas une volonté délibérée de séparation. Cette remarque n'est pas une argutie : elle engage la nature du diagnostic posé sur les questions de ségrégation spatiale et les politiques destinées à y apporter remède.

La seconde clarification, plus centrée sur l'univers de l'enquête, concerne la justice sociale et la mobilité. L'entre soi procède bien d'une tendance socialement ascendantc plutôt que descendante : si tout le monde n'exige pas d'accéder aux meilleures places, personne en tout cas ne souhaite être confronté à des situations inacceptables, régresser en quelque sorte. Tous les parents ne veulent pas s'orienter vers les collèges chics, publics ou privés, mais

3. T. Schelling, par ailleurs prix Nobel d'économie en 2005, a développé depuis 1971 un modèle fondé sur la théorie des jeux qui montre qu'il n'est nul besoin de racisme pour produire un mode d'habitat fortement ségrégué. On trouve aujourd'hui des simulations sur internet qui permettent de faire tourner ce modèle à partir du choix de quelques critères. aucun ne souhaite se diriger vers le ghetto; chacun considère comme injuste d'avoir à se sacrifier au nom d'un intérêt général, de surcroît indémontrable. D'ailleurs, un parent qui ne serait pas animé par cette volonté de faire en sorte que son enfant trouve des bonnes conditions d'épanouissement est considéré comme un mauvais parent. Ainsi, le choix de cet instituteur de scolariser son fils, au nom de l'intérêt général et de la règle commune, dans l'un des collèges les plus durs de la ville, est loin d'être valorisé par ses collègues : "Les militants qui disent qu'il faut laisser leur gamin dans ces écoles, ce sont des fous ou des abrutis ». Ainsi la mixité sociale peut être valorisée positivement à condition de ne pas apparaître comme une perte de chances scolaires.

À chaque fois que l'écart entre les parents favorisés minoritaires et les publics défavorisés majoritaires du secteur devient trop important, les parents ont le sentiment de commettre une mauvaise action s'ils ne transgressent pas. Exercer un tel choix est alors ressenti comme une recherche de la justice pour leur enfant. C'est ce que nous dit ce père d'origine marocaine pour expliquer sa détermination à intervenir dans la scolarité de ses enfants : "rien que d'être dans le collège, en tant que parent, tu es tout de suite classé nul. Je ne veux pas que les profs me prennent pour un idiot parce que j'ai laissé ma fille dans ce collège. En fait, si tu n'es pas un paumé, il faut partir ".

La discrimination ethnique et sociale, si réelle soitelle, ne suffit pas non plus pour rendre compte du mécanisme d'évitement de certains collèges. Ce n'est pas la même chose d'éviter un secteur pour les seules caractéristiques ethniques des publics, ou parce qu'on anticipe un préjudice scolaire, une perte de chances. Dans une école au public socialement très bas et quasi exclusivement d'origine immigrée ou gitane, le directeur s'exprime sans langue de bois : «Ici j’ai deux CM2, 100 \% Maroc, quelques blacks et quelques gitans. Un jour un papa militaire est venu pour inscrire son enfant, il n'avait aucune marque dans la ville. Je lui ai conseillé de repartir et d'aller ailleurs. En arriver à dire ça franchement j'aurais jamais cru. Mais un petit blanc et soixante marocains, ce n'est pas ça la mixité ».

Si les ressources et les stratégies diffèrent selon les catégorics socialcs, tous lcs parcnts sont d'accord sur un point : réussite scolaire attendue et environnement par les pairs conditionnent le jugement porté sur un établissement. À partir de cette base commune, l'action menée par chacun dépend des ressources familiales et du collège proposé.

Les parents sont des acteurs d'une société réflexive : ils connaissent le monde, ils sont informés des sources des inégalités. Si la question de la justice sociale devient à ce point centrale, c'est sous l'effet de l'extension du champ de définition de la notion d'égalité, évolution à laquelle l'école a bien sûr largement contribué. On peut ainsi paradoxalement parler d'inégalités démocratiques, dans 
le sens où elles se dévoilent au fur et à mesure que l'horizon de l'égalité s'élargit. Ainsi le sentiment d'inégalité a une dimension fortement cognitive : la connaissance des mécanismes responsables des places sociales entraîne bien de nouveaux sentiments d'injustice (Meuret, Duru-Bellat, 2009). Ces sentiments - que l'on ne peut liquider en invoquant l'irrationalité ou le racisme - sont aujourd'hui plus intelligibles par la connaissance, même approximative, qu'ont les acteurs du poids des fréquentations plus que par les variables d'appartenance sociale.

\section{Une politique de mixité}

Une politique tenant compte du poids de la mixité devra sans doute modifier le regard de l'institution sur les processus de la différenciation sociale. Jusque-là, la différence sociale ou culturelle des publics est conçue de deux façons : soit à travers un héritage durkheimien comme devant aboutir à une fusion dans un grand corps social moyen, et c'est pourquoi le terme mixité équivaut souvent à neutralité ou normalité ; soit à travers un héritage bourdieusien, construite comme handicap dans un système de domination sociale. C'est pour cette raison que les politiques d'attractivité menées, souvent en catimini, par les établissements autour d'une offre de sections ou d'options, font l'objet de multiples critiques; de même que l'intrusion explicite des choix parentaux dans les politiques scolaires qualifiés de «parentocratie » ou de «marché scolaire ".

Pour un collège, le niveau scolaire, l'image, l'ambiance, l'exposition aux apprentissages, la capacité d'attraction visà-vis des parents et des personnels sont largement fonction du public scolarisé. Cette ressource que constitue le public, difficile à mesurer mais bien réelle, varie sensiblement d'un collège à un autre. Le mélange social caractérise certains lieux (les collèges publics de centre-ville), est beaucoup plus limité dans d'autres (le périurbain public et le privé) voire inexistant comme dans les quartiers difficiles (le ghetto public).

On ne peut plus sc contcntcr aujourd'hui d'cn appcler à un retour à la carte scolaire ou à un encadrement renforcé des dérogations. Ne serait-ce que par l'existence du secteur privé, ces politiques de cantonnement n'ont en rien réussi à endiguer une différenciation des établissements. Pourtant reconnaître la faible efficacité de la sectorisation en termes de mixité ne conduit pas à prôner l'instauration d'une concurrence absolue comme principe général de répartition des élèves dans les collèges. Jusqu'ici les outils politiques mobilisés en France sur cette question sont res- tés de l'ordre de la limitation des mouvements géographiques. Certains pays ont expérimenté d'autres pistes. Il en va, ainsi, de la notion américaine d'accountability (responsabilisation). Selon ce principe, une école ne réussit que si tous les groupes ethniques et sociaux de l'établissement ont atteint des standards prédéfinis (Meuret, 2007).

Une politique de mixité ne pourra occulter les grandes lignes de partage actives dans la distribution géographique des élèves. La première oppose les établissements publics de centre-ville aux établissements des quartiers populaires. La seconde différencie, au sein de la ville centre, le pôle privé et le pôle public. Quant à la troisième, elle oppose les établissements publics du périurbain à ceux de la ville centre. Les établissements du centre-ville ne sont pas les plus élevés socialement, ils sont surtout plus mixtes.

La fracture entre urbain et périurbain, au sein même des collèges publics, constitue une tendance historique forte et la focalisation parisienne et francilienne des études sur la sectorisation scolaire conduit à sous-estimer cette ligne de partage. Les collèges urbains dont la construction est la plus ancienne n'accueillent plus, aujourd'hui les enfants des familles les plus favorisées comme ce fut le cas autrefois. Une bonne part des collèges périurbains plus récents ont aujourd'hui un niveau social plus élevé en même temps qu'une mixité plus faible. Du coup la ville centre se polarise plus fortement entre des quartiers dont les classes moyennes ont émigré vers le périurbain et un centre mixte. Le privé devient alors une valeur refuge des classes moyennes restées dans le centre et qui se voient affectées dans les établissements des quartiers de relégation.

D'autre part les collèges des quartiers difficiles sont plus ségrégués que les quartiers eux-mêmes. Pourtant la force de ce constat ne doit pas conduire à méconnaître la mixité qui persiste dans les collèges du centre. Chercher à établir un système de vases communicants entre les uns et les autres ne conduit qu'à renforcer un secteur privé dont le niveau social moyen s'élève et face auquel aucun établissement du centre ne peut plus rivaliser. Si une politique du ghetto est indispensable tant la ségrégation s'y avère criante, celle-ci ne peut pas tenir lieu de politique de mixitć pour l'cnscmblc d'un bassin de formation. Dans des villes de la taille de celle que nous avons étudiée, le risque est aujourd'hui de perdre la mixité des établissements du centre-ville. Une politique offensive de mixité ne doit pas négliger ces établissements publics qui s'avèrent aujourd'hui les (faibles ?) remparts face à une polarisation toujours plus forte entre le public et le privé comme entre l'urbain et le périurbain. Ces établissements sont mixtes, et l'expérience des élèves et des parents d'une sociabilité complexe et mixte y est encore avérée. 


\section{Références bibliographiques}

Ballion R., (1986), " Le choix du collège : le comportement "éclairé" des familles ", Revue Française de Sociologie, XXVII, pp. 719-734.

Ballion R., Oeuvrard. F., (1991), " Le choix de l'établissement scolaire. Le cas des lycées parisiens ", Éducation et formations, 29. pp. $27-41$.

Caille J.P., (1993), "Le choix d'un collège public situé en dehors du secteur de domiciliation ", Note d'information 93.19, MEN-Direction de l'évaluation et de la prospective.

Coleman J. et al., (1966), Equality of Educational Opportunity, Washington D.C., Government Printing Office.

Combaz G. , (2003), "Les chefs d'établissement face aux paradoxes de l'école démocratique de masse : l'exemple des principaux de collèges publics en France ", Revue des Sciences de l'éducation, vol. $29, n^{\circ} 3$, pp. 629-648.

DEPP, (2003), La carte scolaire et son évitement, Paris, ministère de l’Éducation nationale.

Donzelot J., (2003), Faire société. La politique de la ville aux États-Unis et en France, Paris, Éd. du Seuil.

Donzelot J., (2006), Quand la ville se défait. Quelle politique face à la crise des banlieues?, Paris, Éd. du Seuil.

Dubet F., Martucelli D., (1996), À l'école. Sociologie de l'expérience scolaire,

Paris, Éd. du Seuil.

Dubet F., Duru-Bellat M., (2000), L'hypocrisie scolaire. Pour un collège enfin démocratique, Paris, Éd. du Seuil.

Duru-Bellat M., (2002), Les inégalités sociales à l'école. Genèses et mythe, Paris, Puf.

Duru-Bellat M., Meuret D., (2009), Le sentiment de justice à et sur l'école, Bruxelles, De Boeck.
Felouzis G., Perroton J., Liot F. (2005),

L'apartheid scolaire. Enquête sur la ségrégation ethnique dans les collèges, Paris, Éd. du Seuil.

François J.C., "Évitement à l'entrée en sixième et division sociale de l'espace scolaire à Paris ", L'Espace géographique, $n^{\circ} 4$, pp. 307-327

Lelièvre C., (2004), Les politiques scolaires mises en examen, Paris, ESF.

Maurin E., (2004), Le ghetto français. Enquête sur le séparatisme social, Paris, Éd. du Seuil.

Maurin E., (2007), La nouvelle question scolaire, Paris, Ėd. du Seuil.

MEN-DEP, (2005), "Ségrégation ou mixité : la répartition des élèves dans les collèges et les lycées $"$ Note d'Information, 05.37.

Meuret D., (2007), Gouverner l'école : une comparaison France/États-Unis, Paris, Puf.

Oberti M., (2007), L'école dans la ville.

Ségrégation - Mixité - Carte scolaire,

Paris, Les Presses de Sciences Po.

Prost A., (1979), Histoire de l'enseignement en France, 1800-1967, Paris, Armand Colin.

Rawls J., (1971), Théorie de la justice, Paris, Seuil.

Van Zanten A., (2001), L'école de la périphérie. Scolarité et ségrégation en banlieue, Paris, Puf.

Van Zanten A., Darchy-Koechlin B., (2005), "La formation des élites », Revue internationale d'éducation, $n^{\circ} 39$

Van Zanten A., Obin J.P., (2008), La carte scolaire et le territoire urbain, Paris, Puf.

Visier L., Zoïa G., (2008), La carte scolaire et le territoire urbain, Paris, Puf.

Zoia G., Visier L., (2007). Mixité sociale au collège, Rapport de recherche au Puca, ministère de l'Écologie, du développement et de l'aménagement durables, Paris.

\section{Biographies}

LAURENT VISIER est sociologue, chercheur au CADIS (EHESS Paris) et maître de conférences à I'Université Montpellier 1. II travaille sur la mixité sociale, l'éducation et la santé. II a publié récemment avec Geneviève Zoïa, La carte scolaire et le territoire urbain, avec une préface de François Dubet, Paris, Puf, 2008 et avec Jean-Marc Mouillie, Céline Lefève Médecine et sciences humaines. Manuel pour les études médicales, avec une préface de Tzvetan Todorov, Paris, Les belles lettres, 2007

laurent.visier@univ-montp1.fr

GENEVIEVE ZOÏA est anthropologue, maître de conférences à l'Université Montpellier 2 (IUFM-LIRDEF). Elle travaille sur les questions d'ethnicité dans le champ scolaire. Elle a publié en 2008 avec Laurent Visier, La carte scolaire et le territoire urbain, avec une préface de François Dubet Paris, Puf ; et en 2004, L'accueil des élèves primo-arrivants en France Paris, La documentation française/ FASILD.

zoia@wanadoo.fr 